\title{
Fetal nasal bone hypoplasia in the second trimester and risk of abnormal karyotype in a population of Chinese pregnant women
}

\author{
Yan Du${ }^{1}$, Yunyun Ren ${ }^{2}$, Yingliu Yan ${ }^{2}$, Zhongxing Fu ${ }^{3}$, Chao Zhu ${ }^{3}$ and Li Cao ${ }^{2 *}$ \\ ${ }^{1}$ Office of Clinical Epidemiology, Obstetrics and Gynecology Hospital of Fudan University, Shanghai, China \\ ${ }^{2}$ Ultrasound Department, Obstetrics and Gynecology Hospital of Fudan University, Shanghai, China \\ ${ }^{3}$ Ningguo Bio-Leader Biotechnology Co., LTD., Anhui 242300, China
}

\begin{abstract}
Introduction: To evaluate the association of hypoplastic nasal bone and fetal chromosomal abnormalities in a prescreened sample of Chinese pregnant women.

Materials and methods: This retrospective study followed women whose fetuses shown hypoplastic nasal bone. Fetal karyotyping was performed and pregnancy outcomes were followed. The relationship between hypoplastic nasal bone with abnormal karyotype was evaluated stratified by whether other ultrasound soft markers or structural abnormalities were also observed.

Results: A total of 56707 singleton pregnancies were initially included. After exclusion of unqualified cases, a total of 65 (65/56707, $1.15 \%)$ fetuses with hypoplastic nasal bone were included in the analyses, among which $8(8 / 65,12.31 \%)$ were determined to have chromosomal abnormalities. Hypoplasia of nasal bone in association with other structural abnormalities had a higher rate of abnormal karyotypes $\left[66.67 \%(6 / 9)\right.$ vs. $9.09 \%(2 / 22)$, Fisher's exact test $\left.\chi^{2}=8.256 p=0.004\right]$ compared with cases associated with other ultrasound soft markers.
\end{abstract}

Conclusion: Hypoplastic nasal bone, when associated with structural abnormalities in a fetus, is associated with chromosomal abnormalities.

\section{Introduction}

Ultrasound screening for fetal chromosomal abnormalities is an important component of prenatal diagnosis. Abnormal facial features, such as collapse of the nose bridge, ususally indicate fetal chromosomal abnormalities. There are a number of studies suggesting that fetal nasal bone hypoplasis is an indicator of Down syndrome with relatively high sensitivity and specificity [1-4]. It is reported that the positive likelihood ratio of abnormal fetal nasal bone in screening for Down sydrome was about 11.6-50.5 [2,5-7]. However, most of the above studies were conducted in high risk populations. Few studies have been conducted in samples representative of the general population. In most parts of China, screening for fetal structural abnormalities during second trimester of pregnancy has become routine. Therefore, it is possible to include fetal nasal bone observation and measurement as a routine examination.

In this study, we aimed to evaluate the association between fetal nasal bone hypoplasia and fetal chromosomal abnormalities in a sample of Chinese pregnant women.

\section{Materials and methods}

\section{Study population}

The current study was conducted at the Obstetrics and Gynecology Hospital of Fudan University in Shanghai, China. Our study protocol was approved by the institutional review board of the Hospital, and oral informed consent was obtained from all participants. We included all pregnant women who received prenatal screening and also gave birth or induced labor at our hospital. During January 2012 to December 2015 , there were a total of 58001 births at our hospital, including 1015 twins and other multiple births. In another 279 cases, pregnancies were terminated mid-to-late gestation without receiving prior invasive diagnostic procedures. Thus, we included a total of 56707 singleton pregnancies in the analyses.

\section{Ultrasonographic examinations}

Detailed procedures of the ultrasonographic examinations were published previously [8]. Briefly, Down syndrome screening was carried out at 15-18 gestational weeks of age by a serum markers screening method. High-risk women, including those with advanced maternal age ( $\geq 35$ years) or those classified by the screening test, were counseled for invasive prenatal diagnostic procedures. Second trimester ultrasound scan was performed at 20-23 + six weeks of gestation, using GE Voluson E8 system (GE Medical Systems, Milwaukee, WI, USA), GE Voluson E6 (General Electric Medical System, Milwaukee, WI, USA), or Philips iU 22 (Philips, USA) system equipped with 2-5 MHz transducers.

Correspondence to: $\mathrm{Li}$ Cao, MD, MS, Ultrasound Department, Obstetrics and Gynecology Hospital of Fudan University, No. 419 Fangxie Road, Shanghai, 200011, China, Tel: 8613585891083; E-mail: caolidoc@foxmail.com

Key words: hypoplasia of nasal bone, karyotype abnormality, ultrasound, prenatal screening, down syndrome

Received: November 20, 2017; Accepted: December 08, 2017; Published: December 11, 2017 
Certified physician sonologists carried out the examination according to guidelines of the International Society of Ultrasound in Obstetrics \& Gynecology (ISUOC) [9]. Ultrasound soft markers included: absence or hypoplasia of nasal bone, increased nuchal fold thickness ( $\geq 6 \mathrm{~mm})$, short femur or humerus, ventriculomegaly $(\geq 10 \mathrm{~mm})$, intestinal hyperechogenicity, choroid plexus cyst, echogenic intracardiac focus, mild pyelectasis ( $\geq 5 \mathrm{~mm}$ ), aberrant right subclavian artery, absence of the middle phalanx of the fifth digit and short mandible.

Two-dimensional images of the fetal head in the sagittal plane were used to assess fetal nasal bone length. Images were enlarged to include nose, lips, maxilla and mandibula with an approximately 45 degrees or 135 degrees angle between the insonation beam and the nasal axis to more sharply define the edges of the nasal bone (Figure 1). Three measurements of the fetal nasal bone length were taken, and the largest value was recorded. Measurements of nasal bone length less than the $2.5^{\text {th }}$ percentiles [10], as well as those cases with unilateral absence of nasal bone, were considered short. Pregnant women suspected of abnormal fetal nasal bone were further evaluated by senior sonologists.

Amniocentesis was performed once the diagnosis was confirmed, quantitative fluorescence polymerase chain reaction and karyotyping were carried out to detect for chromosomal abnormalities. Since January 2015, human whole genome single nucleotide polymorphism (SNP) genotyping was applied to evaluate cases with additional risk factors. Neonatal pediatricians observed general situations and clinical signs of those newborns that did not receive prenatal invasive diagnostic procedures. In addition, family history of neonates was considered.

\section{Statistical analyses}

Median with range was summarized for continuous variables, and frequency or rate was calculated for categorical variables. Differences between groups were compared by Chi-square test or Fisher's exact test when appropriate. All analyses were performed by SPSS 16.0 for Windows (SPSS Inc., Chicago, IL, USA). All significance tests were two sided; $p$ value of less than 0.05 was considered as statistically significant.

\section{Results}

Of the 56707 fetus examined, 73 presented abnormal nasal bone development at second trimester ultrasound assessment. Of those

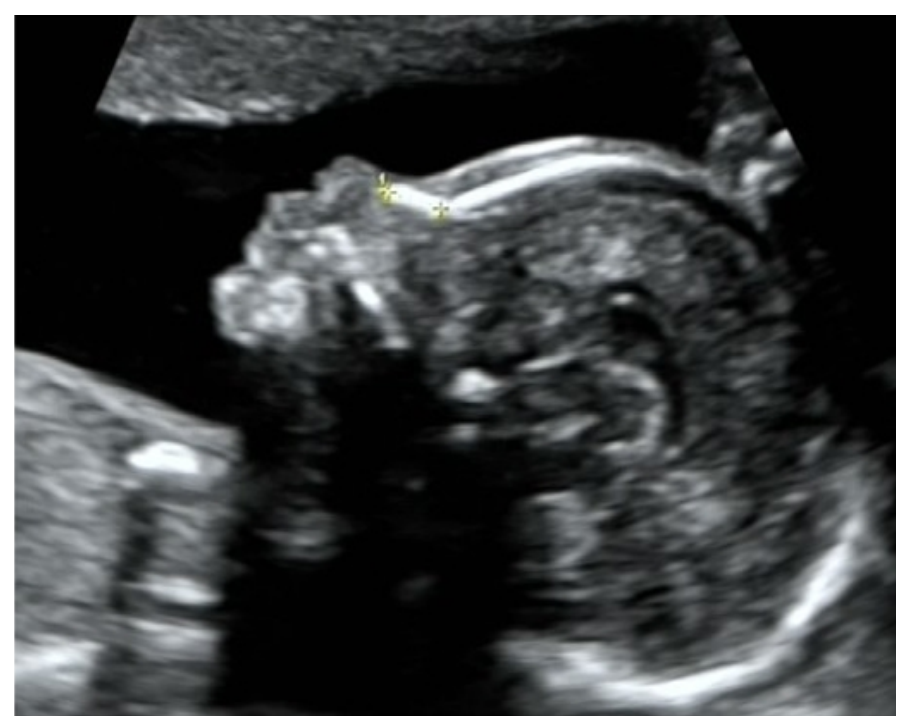

Figure 1. Ultrasound image of fetal nasal bone, showing normal fetal profile at 23 gestational weeks of age
73 cases, three had termination of pregnancy because of multiple structural abnormalities and did not receive genetic amniocentesis, three were confirmed as measurement errors by senior sonologists on further assessment, one underwent termination of pregnancy and did not test for karyotype, and one were one of the twins. Thus, a total of 65 cases with hypoplastic nasal bone were included for final analyses. The prevalence rate of hypoplastic nasal bone was $1.15 \%(65 / 56707)$ in our study population.

The median maternal age was 30 (range: 24-43) years of the 65 pregnant women with hypoplastic fetal nasal bone, and the median gestational weeks at the diagnosis was 22.4 (range: 18.2-23.7) weeks. Of those 65 pregnant women, 50 underwent genetic amniocentesis (76.92\%, 50/65). For those 15 cases that did not undergo amniocentesis, no signs of Down syndrome were observed during postnatal examination and thus were considered normal. There was a total of 8 cases $(12.31 \%, 8 / 65)$ diagnosed to have chromosomal abnormalities, including 5 cases of trisomy-21 (62.50\%), one trisomy-18 (12.50\%), one Klinefelter syndrome (47, XXY) (12.50\%), and one case of microdeletions (in 4p) (12.50\%) (Table 1 and Figure 2).

Among those 65 cases of nasal bone hypoplasia, 34 were isolated nasal bone hypoplasia cases, 22 had other soft ultrasound markers, and nine cases were with additional structural abnormalities (Table 2). The karyotype was normal in 34 cases of isolated nasal bone hypoplasia. There were no differences in chromosomal abnormality rates between isolated hypoplastic fetal nasal bone group and nasal bone hypoplasia with other ultrasound soft marker group [0\% (0/34) vs. 9.09\% (2/22), $\left.\chi^{2}=1.109 p=0.292\right]$ (Table 2). A higher rate of abnormal karyotype [66.67\% (6/9) vs. 9.09\% (2/22), Fisher's exact test $\left.\chi^{2}=8.256 p=0.004\right]$ was observed among fetuses with hypoplastic nasal bone detected in association with structural abnormalities (Table 2).

Figure 3 displays nasal bone measurements in chromosomal abnormalities across gestational age.

\section{Discussion}

It is suggested that hypoplastic nasal bone may be caused by nasal bone hypoplasia or delayed ossification. Studies have shown that the normal ranges of second trimester fetal nasal bone length vary significantly among different ethnic groups [11]. Sonek, et al. [12] using the definition of nasal hypoplasia when the nasal bone length is below $3 \mathrm{~mm}$ at 16 weeks and $4.5 \mathrm{~mm}$ at 20 weeks since the $2.5^{\text {th }}$ percentiles and $5^{\text {th }}$ percentiles in the second trimester are quite consistent across studies, reported that the occurrence of absent or hypoplastic fetal nasal bone was $3.66 \%$ in their pregnant population at $15-24$ weeks of gestation, and the incidence of abnormal nasal bone among Down syndrome cases was $60 \%$. In our study, using $2.5^{\text {th }}$ percentiles, the rate of hypoplastic fetal nasal bone was only $1.15 \%$, lower than other studies $(4.34 \%-43.48 \%)$ [13-15], possibly because the current study was carried out in a prescreened sample with lower risk than the general population, while other studies reported results from high-risk populations. This discrepancy may also be related to ethnic differences. There might be observer variance in our study. The exact length of the fetal nasal bone would be measured when hypoplastic nasal bone was suspected by the observer. So, we cannot rule out the possibility of observer's subjective factors when determining short fetal nasal bone; and it is likely some fetuses with slight short nasal bone or close to the cut-off value might be missed out. To improve this shortcoming, we are planning a prospective study which will measure the exact length of each fetal nasal bone to establish the range of normal fetal nasal bone length during second trimester in our region. However, our 
Table 1. Summary of the cases identified with chromosomal abnormalities by genetic amniocentesis $(n=24)$

\begin{tabular}{|c|c|c|c|c|c|}
\hline Case Number & $\begin{array}{l}\text { Maternal age } \\
\text { (Year) }\end{array}$ & $\begin{array}{l}\text { Gestational age } \\
\text { (week) }\end{array}$ & $\begin{array}{l}\text { Ultrasound marker of } \\
\text { nasal bone }\end{array}$ & Other ultrasound markers & Embryonic karyotype \\
\hline 1 & 34 & 22.3 & Nasal bone length $=3.9 \mathrm{~mm}$ & Short femur, small head circumference & Trisomy-21 \\
\hline 2 & 32 & 23.5 & Nasal bone length $=4.1 \mathrm{~mm}$ & Enlarged posterior fossa & Trisomy-21 \\
\hline 3 & 32 & 20.4 & Nasal bone length $=3.4 \mathrm{~mm}$ & $\begin{array}{l}\text { Short femur, strawberry-shaped skull, absence of septum } \\
\text { pellucidum, bilateral choroid plexus cyst, short mandible, large } \\
\text { ventricular septal defect, bilateral overriding fingers }\end{array}$ & Trisomy-18 \\
\hline 4 & 27 & 22.3 & Nasal bone length $=3.8 \mathrm{~mm}$ & Small cerebellum & $\begin{array}{l}\text { Klinefelter syndrome } \\
(47, \mathrm{XXY})\end{array}$ \\
\hline 5 & 38 & 22.6 & Nasal bone length $=3.9 \mathrm{~mm}$ & $\begin{array}{l}\text { Fetal growth retardation, possible tracheoesophageal fistula, } \\
\text { complex congenital heart disease }\end{array}$ & $\begin{array}{l}\text { Micro-deletion (arr4p16.3 } \\
\text { p16.1×1) }\end{array}$ \\
\hline 6 & 38 & 22.6 & Nasal bone length $=3.9 \mathrm{~mm}$ & Possible tracheoesophageal fistula & Trisomy-21 \\
\hline 7 & 42 & 23.1 & Nasal bone length $=3.4 \mathrm{~mm}$ & $\begin{array}{l}\text { Tetralogy of Fallot, absence of corpus callosum, increased } \\
\text { nuchal fold thickness }\end{array}$ & Trisomy-21 \\
\hline 8 & 43 & 23.6 & Nasal bone length $=2.6 \mathrm{~mm}$ & Duodenal stenosis or atresia, hydramnios & Trisomy-21 \\
\hline
\end{tabular}

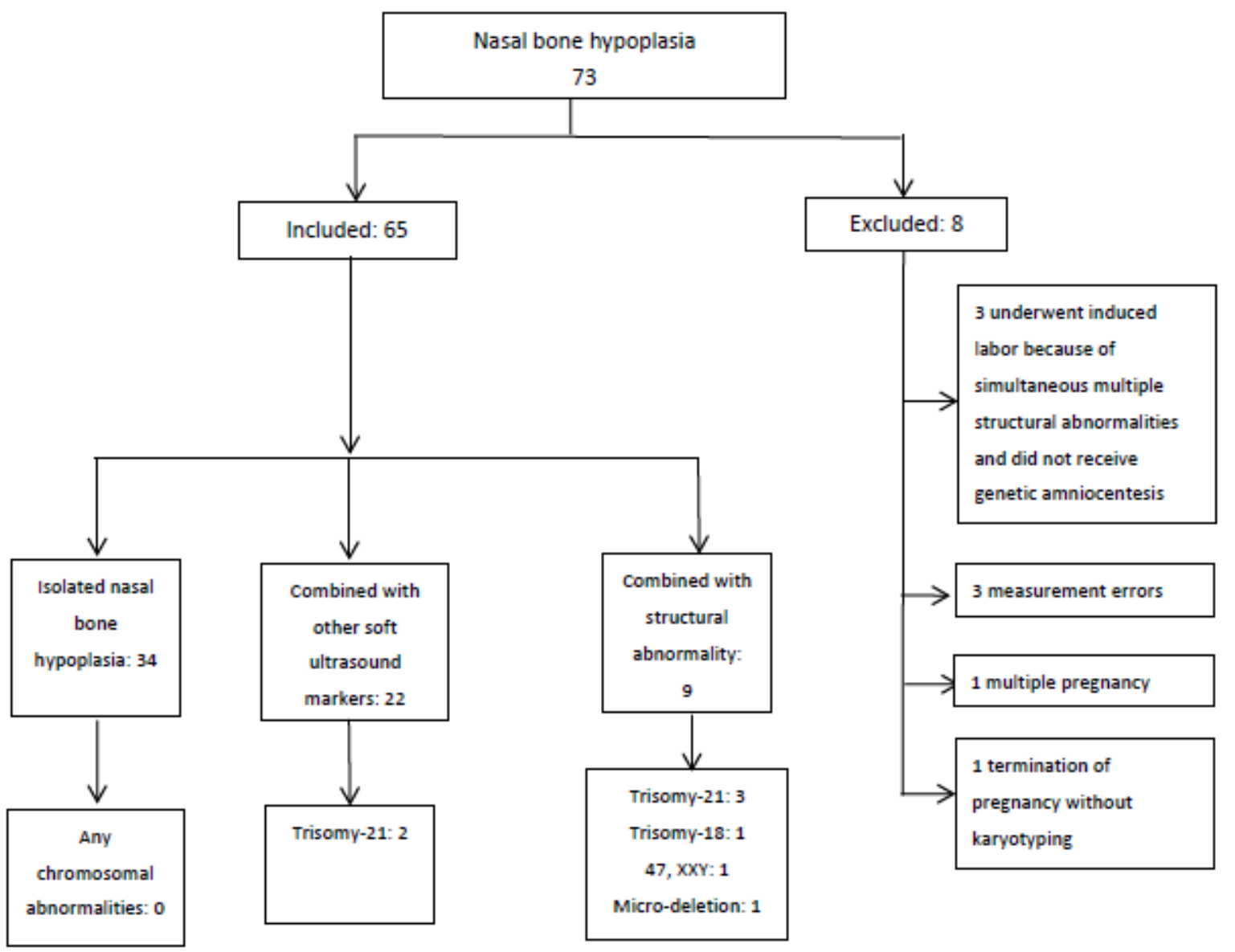

Figure 2. Characterization of 65 cases with nasal bone hypoplasia

Table 2. Number and rate of chromosomal abnormalities associated with abnormal nasal bone (aNB)

\begin{tabular}{|l|l|l|l|}
\hline \multirow{2}{*}{$\begin{array}{l}\text { Type of chromosomal } \\
\text { abnormalities }\end{array}$} & \multicolumn{3}{|l|}{ NB hypoplasia (n=65) } \\
\cline { 2 - 4 } & $\begin{array}{l}\text { Isolated hNB } \\
(\mathrm{n}=34)\end{array}$ & $\begin{array}{l}\text { hNB+other } \\
\text { marker (n=22) }\end{array}$ & $\begin{array}{l}\text { hNB+ structural } \\
\text { abnormalities (n=9) }\end{array}$ \\
\hline Trisomy-21 (n, \%) & $0(0 \%)$ & $2(9.09 \%)$ & $3(33.33 \%)$ \\
\hline Trisomy-18 (n, \%) & $0(0 \%)$ & $0(0 \%)$ & $1(11.11 \%)$ \\
\hline $\mathbf{4 7 , ~ X X Y ~ ( n , ~ \% ) ~}$ & $0(0 \%)$ & $0(0 \%)$ & $1(11.11 \%)$ \\
\hline Micro-deletion (n, \%) & $0(0 \%)$ & $0(0 \%)$ & $1(11.11 \%)$ \\
\hline Total abnormalities (n, \%) & $0(0 \%)$ & $2(9.09 \%)$ & $6(66.67 \%)$ \\
\hline Normal/Presumed normal (n, \%) & $34(100 \%)$ & $20(90.91 \%)$ & $3(33.33 \%)$ \\
\hline
\end{tabular}

hNB: Hypoplastic nasal bone study protocol strictly follows ISUOC guidelines and the sonologists were rigorously trained. Standard image of each fetal nasal bone was taken and stored, every three months a senior sinologist (Yingliu Yan) would randomly select 20-30 images of each sonologist to review for quality control purpose. In addition, each abnormal fetal nasal bone case detected during second trimester ultrasound examination would be further evaluated by senior sinologists to ensure the accuracy of the final diagnosis of abnormal fetal nasal bone development.

The current study observed no chromosomal abnormalities among fetuses with isolated hypoplastic nasal bone development; while additional soft ultrasound markers were present, the detection rate was 


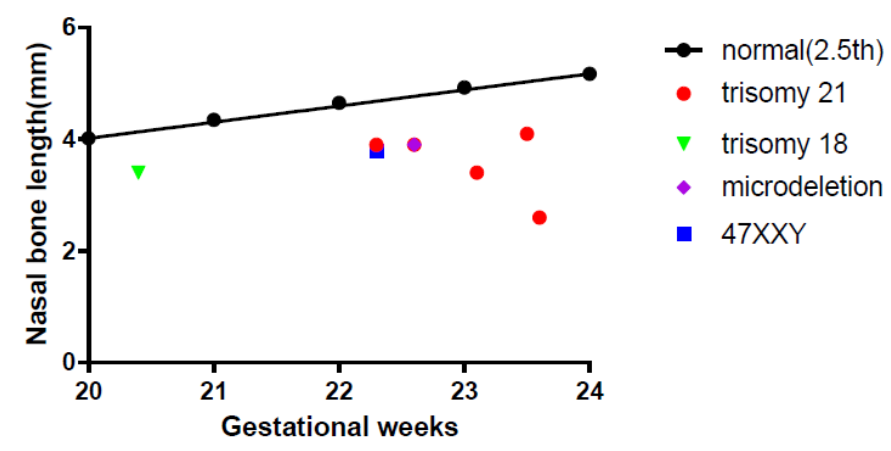

Figure 3. Nasal bone measurements in chromosomal abnormalities across gestational age

$9.09 \%(2 / 22)$. When hypoplastic nasal bone was presented in association with structural abnormalities, the detection rate increased to $66.67 \%$ (6/9). Therefore, although the prognosis of isolated hypoplastic fetal nasal bone was relatively good, a detailed fetal morphology scan is still necessary when hypoplastic fetal nasal bone is detected at the second trimester ultrasound screening.

Abnormal fetal nasal bone development may be associated with multiple chromosomal abnormalities [16-18]. The most common fetal chromosomal abnormality detected in the current study was Down syndrome $(62.5 \%, 5 / 8)$, other chromosomal abnormalities were Trisomy-18 (3 cases), 47, XXY (1 case), and micro-deletion (1 case). Human whole genome SNP genotyping has been routinely applied for amniocentesis in our hospital just about two years ago, and it is possible that there was an under-estimation of chromosomal micro-deletions or micro-duplications. Currently our hospital has recommended gene sequencing of newborns with collapsed nose bridge, and long time follow-up of the growth and development conditions of these children is also very important.

This retrospective study did not measure nasal bone length of all fetuses, therefore, we cannot rule out the possibility of false negative cases to bias our results. In addition, karyotyping could not be performed on 8 fetuses with abnormal fetal nasal bone due to various reasons, which may also affect our study results.

In conclusion, hypoplastic nasal bone in fetus, in combination with structural abnormalities, is strongly associated with chromosomal abnormalities, whereas isolated nasal bone hypoplasia is not. Detailed prenatal diagnosis is advisable to rule out abnormal karyotype when hypoplastic nasal bone is detected.

\section{Conflicts of interest}

The authors have declared that they have no conflicts of interests in connection with this article.

\section{References}

1. Moreno-Cid M, Rubio-Lorente A, Rodríguez MJ, Bueno-Pacheco G, Tenías JM, et al. (2014) Systematic review and meta-analysis of performance of second-trimester nasal bone assessment in detection of fetuses with Down syndrome. Ultrasound Obstet Gynecol 43: 247-53.

2. Agathokleous M, Chaveeva P, Poon LC, Kosinski P, Nicolaides KH (2013) Metaanalysis of second-trimester markers for trisomy 21. Ultrasound Obstet Gynecol 41 : 247-261

3. Shanks A, Odibo A (2010) Nasal bone in prenatal trisomy 21 screening. Obstet Gynecol Surv 65: 46-52.

4. Driscoll DA, Gross S (2009) Clinical practice. Prenatal screening for aneuploidy. $N$ Engl J Med 360: 2556-2562.

5. Dukhovny S, Wilkins-Haug L, Shipp TD, Benson CB, Kaimal AJ, et al. (2013) Absent fetal nasal bone: what does it mean for the euploid fetus? J Ultrasound Med 32: 2131-2134.

6. Kagan KO, Cicero S, Staboulidou I, Wright D, Nicolaides KH (2009) Fetal nasal bone in screening for trisomies 21,18 and 13 and Turner syndrome at 11-13 weeks of gestation. Ultrasound Obstet Gynecol 33: 259-264. [Crossref]

7. Stressig R, Kozlowski P, Froehlich S, Siegmann HJ, Hammer R, et al. (2011) Assessment of the ductus venosus,tricuspid blood flow and the nasal bone in secondtrimester screening for trisomy 21. Ultrasound Obstet Gynecol 37: 444-449.

8. Du Y, Ren Y, Yan Y, Cao L (2017) Absent fetal nasal bone in the second trimester and risk of abnormal karyotype in a prescreened population of Chinese women. Acta Obstetricia et Gynecologica Scandinavica (AOGS). [Crossref]

9. International Society of Ultrasound in Obstetrics \& Gynecology (2016) Cardiac screening examination of the fetus: guidelines for performing the 'basic' and 'extended basic' cardiac scan. Ultrasound Obstet Gynecol 27: 107-113.

10. Hung JH, Fu CY, Chen CY, Chao KC, Hung J (2008) Fetal nasal bone length and Down syndrome during the second trimester in a Chinese population. J Obstet Gynaecol Res 34: 518-523.

11. Papasozomenou P, Athanasiadis AP, Zafrakas M, Panteris E, Loufopoulos A, et al (2016) Fetal nasal bone length in the second trimester: comparison between population groups fromdifferent ethnic origins. J Perinat Med 44: 229-235.

12. Sonek JD, Cicero S, Neiger R, Nicolaides KH (2006) Nasal bone assessment in prenatal screening for trisomy 21. Am J Obstet Gynecol 195: 1219-1230.

13. Cusick W, Shevell T, Duchan LS, Lupinacci CA, Terranova J, et al. (2007) Likelihood ratios for fetal trisomy 21 based on nasal bone length in the second trimester: how best to define hypoplasia? Ultrasound Obstet Gynecol 30: 271-274.

14. Gianferrari EA, Benn PA, Dries L, Brault K, Egan JF, et al. (2007) Absent or shortened nasal bone length and the detection of Down Syndrome in second-trimester fetuses. Obstet Gynecol 109: 371-375. [Crossref]

15. Persico N, Borenstein M, Molina F, Azumendi G, Nicolaides KH (2008) Prenasal thickness in trisomy-21 fetuses at 16-24 weeks of gestation. Ultrasound Obstet Gynecol 32: 751-754.

16. Kagan KO, Sonek J, Berg X, Berg C, Mallmann M, et al. (2015) Facial markers in second- and third-trimester fetuses with trisomy 18 or 13, triploidy or Turner syndrome. Ultrasound Obstet Gynecol 46: 60-65.

17. Ting YH, Lao TT, Lau TK, Chung MK, Leung TY (2011) Isolated absent or hypoplastic nasal bone in the second trimester fetus: is amniocentesis necessary? J Matern Fetal Neonatal Med 24: 555-558. [Crossref]

18. Yang X, Han J, Zhen L, Pan M, Li D, et al. (2015) Relationship between absent or hypoplastic fetal nasal bone and chromosome abnormalities: analysis of 187 cases. Chin J Perinat Med 18: 339-342.

Copyright: (C2017 Du Y. This is an open-access article distributed under the terms of the Creative Commons Attribution License, which permits unrestricted use, distribution, and reproduction in any medium, provided the original author and source are credited. 